\title{
ANALYTIC HYPOELLIPTICITY OF CERTAIN SECOND-ORDER EVOLUTION EQUATIONS WITH DOUBLE CHARACTERISTICS
}

BY

\author{
MARIO TOSQUES
}

\begin{abstract}
The present article establishes the analytic hypoellipticity (Definition 1.2) of a class of abstract evolution equations of order two, with double characteristics, under the hypothesis that the coefficients are analytic (in a suitable sense; see $\$ 2$ ). The noteworthy feature of the main result (Theorem 4.1) is that analytic hypoellipticity holds whenever hypoellipticity does, even when one of the asymptotic eigenvalues $c^{j}(A)$ fails to be elliptic of order one.
\end{abstract}

Introduction. The present paper establishes the analytic hypoellipticity (Definition 1.2) of a class of (abstract) evolution equations of order two, with double characteristics-precisely the same class as that studied in the work [1], but under the hypothesis that the coefficients are analytic (in a suitable sense; see $\S 2$ ). We adapt the concatenation method of [1] and use the method of Grushin [2] to derive analytic hypoellipticity from suitable a priori estimates. The noteworthy feature of the main result (Theorem 4.1) is that analytic hypoellipticity holds whenever hypoellipticity does (under the analyticity hypothesis concerning the coefficients)-even when one of the asymptotic eigenvalues $c^{j}(A)$ fails to be elliptic of order one (in the latter case our results are essentially particular cases of those of Grushin). Since the equations studied here are microlocal models for the pseudodifferential equations of the kind studied in [4], our main result strongly suggests that, when their total symbols are analytic, the operators II studied in [4] are hypoelliptic analytic if and only if all their asymptotic eigenvalues are.

I wish to thank Professor F. Treves for suggesting the problem and his contributions to many of the arguments.

1. Analytic hypoellipticity in the abstract set-up. Let $A$ be a linear operator, densely defined in a Hilbert space $H$. We shall assume that $A$ is unbounded but it is selfadjoint, positive-definite and that it has a bounded inverse $A^{-1}$.

Let $J$ be a given open subset of the real line. We denote by $2_{A}(J)$ the ring of the series in the nonnegative powers of $A^{-1}$ with coefficients in $C^{\infty}(J)$ which converge in $L(H, H)$ (the Banach space of bounded linear operators of $H$ into $H$ )

Received by the editors August 1, 1974.

AMS (MOS) subject classifications (1970). Primary 35H05, 35 S99.

Key words and phrases. Evolution equation, hypoelliptic, analytic hypoelliptic, concatenation, scale of Hilbert spaces. 
as well as each one of their derivatives in $t$, uniformly with respect to $t$ on compact subsets of $J$.

$2_{A}((J))$ will denote the formal power series in $A^{-1}$, with coefficients in $C^{\infty}(J)$

Now we want to state precisely what we mean for analytic hypoellipticity of an operator of the kind

$$
P=\sum_{J+k=m} a_{J, k}(t, A) A^{J} \partial_{t}^{k}
$$

where $a_{J, k}(t, A) \in Q_{A}(J)$. Then we have to introduce a scale of "Sobolev spaces" $H^{s}, s \in \mathbf{R}$, defined by $A$ :

If $s \geqslant 0, H^{s}$ is the space of elements $u \in H$ such that $A^{s} u \in H$, equipped with the norm $\|u\|_{s}=\left\|A^{s} u\right\|_{0}$, where \|\|$_{0}$ denotes the norm in $H$.

If $s<0, H^{s}$ is the completion for the norm $\|u\|_{s}=\left\|A^{s} u\right\|_{0}$.

The inner product in $H^{s}$ will be denoted by $(,)_{s}$.

Whatever $s, m \in \mathbf{R}, A^{m}$ is an isomorphism (for the Hilbert space structure) of $H^{s}$ onto $H^{s-m}$.

We denote by $H^{\infty}$ the intersection of the spaces $H^{s}$ and by $H^{-\infty}$ their union, the former equipped with the projective limit topology and the latter with the inductive limit topology.

$H^{\infty}$ and $H^{-\infty}$ with their topology are the strong dual of each other as $H^{s}$ and $H^{-s}$ are.

We denote by $C^{\infty}\left(J, H^{\infty}\right)$ the space of the $C^{\infty}$ functions in $J$ valued in $H^{\infty}$, equipped with its natural $C^{\infty}$ topology. It is the intersection of the spaces $C^{h}\left(J, H^{k}\right)$ as $h$ and $k$ tend to $\infty$, of the $h$-continuously differentiable functions defined in $J$ and valued in the Hilbert space $H^{k}$.

If $K$ is any compact subset of $J$, we denote by $C_{c}^{\infty}\left(K, H^{\infty}\right)$ the subspaces of $C^{\infty}\left(J, H^{\infty}\right)$ consisting of the functions which vanish identically outside of $K$. this is a closed linear subspace of $C^{\infty}\left(J, H^{\infty}\right)$, then a Fréchet space and we denote by $C_{c}^{\infty}\left(J, H^{\infty}\right)$ the inductive limit of the $C_{c}^{\infty}\left(K, H^{\infty}\right)$ as $K$ ranges over the compact subsets of $J$.

Now we can define the space $D^{\prime}\left(J, H^{-\infty}\right)$ of the distributions in $J$ valued in $H^{-\infty}$ as the dual of $C_{c}^{\infty}\left(J, H^{\infty}\right)$.

Finally we denote by $A\left(J, H^{\omega}\right),\left[C^{\infty}\left(J, H^{\omega}\right)\right]$, the subspace of $C^{\infty}\left(J, H^{\infty}\right)$ given by the set of the functions $u(t) \in C^{\infty}\left(J, H^{\infty}\right)$ such that for every point $t_{0} \in J$, there is an open neighborhood $J^{\prime}$ of $t_{0}$ relatively compact contained in $J$ and a constant $C>0$ such that for every $\alpha, \beta \in \mathbf{N}$ 


$$
\begin{aligned}
& \sup _{t \in J^{\prime}}\left\|\partial_{t}^{\alpha} A^{\beta} u(t)\right\|_{0} \leqslant C^{\alpha+\beta+1}(\alpha+\beta) ! \\
& {\left[\sup _{t \in J^{\prime}}\left\|A^{\beta} u(t)\right\|_{0} \leqslant C^{\beta+1} \beta\right] !}
\end{aligned}
$$

Definition 1.1. Let $P$ be as in (1.1), $P$ is said to be hypoelliptic in $J$ if given any open subset $J^{\prime}$ of $J$ and any distribution $u(t) \in D^{\prime}\left(J^{\prime}, H^{-\infty}\right)$,

$$
P u \in C^{\infty}\left(J^{\prime}, H^{\infty}\right) \Rightarrow u \in C^{\infty}\left(J^{\prime}, H^{\infty}\right) .
$$

We say that $\mathbf{P}$ is hypoelliptic at a point $t_{0}$ of $J$ if there is a neighborhood $J^{\prime}$ of $t_{0}$ in $J$ such that $\mathbf{P}$ is hypoelliptic in $J^{\prime}$.

Definition 1.2. $\mathbf{P}$ is said to be analytic hypoelliptic in $J$ if $\mathbf{P}$ is hypoelliptic in $J$ and if, given any open subset $J^{\prime}$ of $J$ and any distribution $u(t) \in D^{\prime}\left(J^{\prime}, H^{-\infty}\right)$,

$$
P u \in \mathrm{A}\left(J^{\prime}, H^{\omega}\right) \Rightarrow u \in \mathrm{A}\left(J^{\prime}, H^{\omega}\right) .
$$

Similarly $\mathbf{P}$ is analytic hypoelliptic at a point $t_{0}$ of $J$ if there is a neighborhood $J^{\prime}$ of $t_{0}$ in $J$ such that $\mathbf{P}$ is analytic hypoellyptic in $J^{\prime}$.

We introduce, now, a new scale of Hilbert spaces.

If $s \in \mathrm{R}$ and $s \geqslant 0$, we denote by $E_{s}$ the set of all elements $u \in H$ for which there is a $v \in H$ such that $u=e^{-s A} v$ and we put in $E_{s}$ the norm $\|u\|_{E_{s}}=\|v\|_{0}$. $\left\|e^{s A} u\right\|_{0}$.

If $s \in \mathbf{R}$ and $s<0, E_{s}$ is the completion of $H$ with the norm $\|u\|_{E_{s}}=$

For every real $s, E_{s}$ is a Hilbert space with the norm \|\|$_{E_{s}}$ and the following properties are true

(i) For every $s$ and $s^{\prime}$ such that $s>s^{\prime}, E_{s}$ is canonically imbedded in $E_{s}$, with a norm $\leqslant 1$.

(ii) For every $s$ and $s^{\prime}$ such that $s>s^{\prime}, A$ defines a continuous linear map $A: E_{s} \rightarrow E_{s^{\prime}}$, with norm $\leqslant C /(s-s)^{\prime}$, where $C$ is a positive constant independent on $s$ and $s^{\prime}$.

For every real $s$, we denote by $A_{t}\left(J, E_{s}\right)$ the space of the analytic functions on $J$ with value in $E_{s}$.

REMARK 1.1. It is easy to see that

$$
A\left(J, H^{\omega}\right)=\bigcup_{s>0} A_{t}\left(J, E_{s}\right) .
$$

2. "Classes" of cut off functions. Let $J$ denote an open interval centered in the origin; we are going to study the analytic hypoellipticity for "formal" operators of the form 


$$
P=\left(\partial_{t}-a(t, A) A\right)\left(\partial_{t}-b(t, A) A\right)-c(t, A) A=X Y-c(t, A) A
$$

where

$$
X=\partial_{t}-a(t, A) A ; \quad Y=\partial_{t}-b(t, A) A
$$

and $a(t, A), b(t, A), c(t, A) \in 2_{A}((J))$ which satisfies the following properties:

If $a_{0}(t)$ and $b_{0}(t)$ are the leading coefficients of $a(t, A)$ and $b(t, A)$, then

$$
a_{0}(0)=b_{0}(0)=0
$$

$$
\operatorname{Re} a_{0}^{\prime}(0)>0, \quad \operatorname{Re} b_{0}^{\prime}(0)<0 .
$$

Furthermore, if we write $a(t, A)=\Sigma_{k} a_{k}(t) A^{-k}, b(t, A)=\Sigma_{k} b(t) A^{-k}$, $c(t, A)=\Sigma_{k} c_{k}(t) A^{-k}$, we suppose that there is a neighborhood $U$ of 0 in $C$, suct that $U \cap \mathbf{R}=J$, on which the $C^{\infty}$ functions $a_{k}(t), b_{k}(t), c_{k}(t)$ can be extended as holomorphic functions satisfying the inequality

$$
\left\{\left|a_{k}(z)\right|,\left|b_{k}(z)\right|,\left|c_{k}(z)\right|\right\} \leqslant c^{k+1} k !
$$

for every integer $k \geqslant 0$ and for every $z \in U$.

Therefore, by Cauchy's inequality, after a shrinking of $U$, we can suppose that there is a constant $M>0$ such that

$$
\left|\partial_{t}^{\alpha} a_{k}(z)\right|,\left|\partial_{t}^{\alpha} b_{k}(z)\right|,\left|\partial_{t}^{\alpha} c_{k}(z)\right| \leqslant c^{k+1} M^{\alpha} \alpha ! k !
$$

for every integer $\alpha, k \geqslant 0$ and for every $z \in U$.

Because $A$ is a selfadjoint operator, using the spectral resolution of $A$, we have that

$$
\begin{aligned}
A & =\int_{-\infty}^{+\infty} \lambda d E_{\lambda}=\int_{\sigma(A)} \lambda d E_{\lambda}, \\
A^{-1} & =\int_{-\infty}^{+\infty} \lambda^{-1} d E_{\lambda}=\int_{\sigma(A)} \lambda^{-1} d E_{\lambda}
\end{aligned}
$$

where $\sigma(A)$ is the spectrum of $A$.

Below $\left\{\varphi_{k}(\lambda)\right\}, k \in \mathbf{N}$, denotes a sequence of continuous real valued functions defined on $\mathbf{R}$.

DEFINITION 2.1. Let $c_{1}$ be a number $>0$. We will say that the sequence $\left\{\varphi_{k}(\lambda)\right\}$ belongs to the "class of cut off functions" $\left[c_{1}\right]$ if

(1) $\varphi_{0}(\lambda)=1$ for every $\lambda \in \mathbf{R}$,

(2) $0 \leqslant \varphi_{k}(\lambda) \leqslant 1$ for every $k \geqslant 1$ and $\lambda \in \mathbf{R}$ and

$$
\varphi_{k}(\lambda)= \begin{cases}1 & \forall \lambda \geqslant c_{1} k+1, \\ 0 & \forall \lambda \leqslant c_{1} k .\end{cases}
$$

Then for every nonnegative integer $k$, the operator 


$$
\varphi_{k}(A)=\int \varphi_{k}(\lambda) d E_{\lambda}
$$

is a bounded selfadjoint linear operator defined on $H$ into $H$.

We define formally

(2.9) $\mathrm{P}\left(\left\{\varphi_{k}\right\}\right)=\left(\partial_{t}-a\left(t, A,\left\{\varphi_{k}\right\}\right) A\right)\left(\partial_{t}-b\left(t, A,\left\{\varphi_{k}\right\}\right) A\right)-c\left(t, A,\left\{\varphi_{k}\right\}\right) A$

where

$$
\begin{aligned}
& a\left(t, A, \varphi_{k}\right)=\sum_{k} a_{k}(t) \varphi_{k}(A) A^{-k}, \\
& b\left(t, A, \varphi_{k}\right)=\sum_{k} b_{k}(t) \varphi_{k}(A) A^{-k}, \\
& c\left(t, A, \varphi_{k}\right)=\sum_{k} c_{k}(t) \varphi_{k}(A) A^{-k} .
\end{aligned}
$$

REMARK 2.1. If $c_{1}>c$, where $c$ is given by (2.4), for every $\left\{\varphi_{k}\right\} \in\left[c_{1}\right]$ $\mathrm{P}\left(\left\{\varphi_{k}\right\}\right)$ is a "true" operator, that is the coefficients $a\left(t, A,\left\{\varphi_{k}\right\}\right), b\left(t, A,\left\{\varphi_{k}\right\}\right)$ and $c\left(t, A,\left\{\varphi_{k}\right\}\right)$ are $C^{\infty}$ functions defined on $J$ with value in $L(H, H)$.

Indeed, take an integer $\alpha$ and let \|\| denote the norm $L(H, H)$; we have, by (2.5),

$$
\begin{aligned}
\sup _{t \in J}\left\|\sum_{0}^{n}\left(\partial_{t}^{\alpha} a_{k}\right)(t) \varphi_{k}(A)^{-k}\right\| \\
\quad \leqslant \sum_{k=0}^{h} \sup _{J}\left|\partial_{t}^{\alpha} a_{k}(t)\right| \cdot\left\|\varphi_{k}(A) A^{-k}\right\| \\
\quad \leqslant \sum_{k=0}^{n} c^{k+1} M^{\alpha} \alpha ! k ! \cdot\left(c_{1} k\right)^{-k} \leqslant c M^{\alpha} \alpha !\left(\sum_{0}^{\infty}\left(\frac{c}{c_{1}}\right)^{k}\right),
\end{aligned}
$$

which proves the result.

Then there is a constant $M_{1}$ such that for every $\alpha \in N$

$$
\begin{aligned}
\sup _{J}\left\{\left\|\partial_{t}^{\alpha} a\left(t, A,\left\{\varphi_{k}\right\}\right)\right\|,\left\|\partial_{t}^{\alpha} b\left(t, A,\left\{\varphi_{k}\right\}\right)\right\|,\left\|\partial_{t}^{\alpha} c\left(t, A,\left\{\varphi_{k}\right\}\right)\right\|\right\} & =M_{1}^{\alpha+1} \alpha !
\end{aligned}
$$

Suppose that $\mathbf{P}_{\mathbf{1}}$ and $\mathbf{P}_{\mathbf{2}}$ are operators of the kind

$$
\mathrm{P}_{1}=\sum_{J+k=2} F_{J, k}(t) A^{J} \partial_{t}^{k}, \quad \mathrm{P}_{2}=\sum_{J+k=2} G_{J, k}(t) A^{J} \partial_{t}^{k}
$$

where $F_{J, k}(t), G_{J, k}(t)$ are analytic functions on $J$ with value in $L(H, H)$ which can be extended as holomorphic functions on the complex neighborhood $U$ of 0 $(J=U \cap \mathbf{R})$ with value in $L(H, H)$ and $F_{0,2}(t)=G_{0,2}(t)=I_{H}, I_{H}$ being the identity mapping of $H$. 
THEOREM 2.1. Let us suppose that

$$
\mathbf{P}_{1}=\mathbf{P}_{2}+\mathbf{R}
$$

where $\mathbf{R}$ is a linear operator which has the following property: there is an $\epsilon>0$ such that for every real s

$$
R: A_{t}\left(J, E_{s}\right) \rightarrow A_{t}\left(J, E_{s+\epsilon}\right)
$$

is a linear operator of $A_{t}\left(J, E_{s}\right)$ into $A_{t}\left(J, E_{s+\epsilon}\right)$.

Then if $\mathbf{P}_{2}$ is analytic hypoelliptic at $t=0$, the same is true of $\mathbf{P}_{1}$.

PROOF. For the proof we will use some results of [3], using the scale of Hilbert spaces $E_{s}$.

Let $u$ be an element of $D^{\prime}\left(J, H^{-\infty}\right)$ such that $\mathbf{P}_{1} u=f$ where $f \in A\left(J, H^{\omega}\right)$, then $f \in A_{t}\left(J, E_{0}\right)$, recalling that $E_{0}=H$, then if we shrink enough the complex neighborhood $U$ of 0 we can extend $f$ to a holomorphic function on $U$ with values in $E_{0}$, i.e. $f \in A_{t}\left(U, E_{0}\right)$.

Now we reduce the second order differential equation in $t, \mathbf{P}_{1} u=f$, to a first order linear system

$$
L U=I \partial_{t} U-M(t, A) U=F(t)
$$

where $I$ is the identity matrix $2 \times 2, M(t, A)$ a $2 \times 2$ matrix and $F(t)$ a twovector, holomorphically dependent on $t$ varying in $U$.

Now for (i) and (ii) of (1.3) the hypothesis of Theorem 2.1 in [3] are verified and by the uniqueness of the solution and by Remark 9.3 in [3], for every $s<0, u$ is the restriction of a holomorphic function $\tilde{u}(t)$ on $U$ (after a shrinking of $U$ ) with value in $E_{s}$, in particular we have $u \in A_{t}\left(J, E_{s}\right)$.

Then by property (2.12), if we choose $s=-\epsilon / 2$, we have that $R u \in$ $A_{t}\left(J, E_{\epsilon / 2}\right)$, then by Remark 1.1 we have that $R u \in A\left(J, H^{\omega}\right)$.

Therefore

$$
\mathbf{P}_{2} u=\mathbf{P}_{1} u-R u \in A\left(J, H^{\omega}\right),
$$

then by the analytic hypoellipticity, at $t=0$, of $P_{2}$ we get that also $P_{1}$ is analytic hypoelliptic at $t=0$.

We have now the following consequence

THEOREM 2.2. Suppose that $c_{1}$ and $c_{2}$ are two constants such that

$$
c_{1}>c, \quad c_{2}>c
$$

where $c$ is the constant given in (2.4), if $\left\{\varphi_{k}\right\} \in\left[c_{1}\right]$ and $\left\{\psi_{k}\right\} \in\left[c_{2}\right]$, then $\mathbf{P}\left(\left\{\varphi_{k}\right\}\right)$ is analytic hypoelliptic at $t=0$ if and only if this is so for $\mathbf{P}\left(\left\{\psi_{k}\right\}\right)$.

Proof. We have 


$$
\begin{aligned}
\mathrm{P}\left(\left\{\varphi_{k}\right\}\right)= & \mathrm{P}\left(\left\{\psi_{k}\right\}-\left\{\psi_{k}-\varphi_{k}\right\}\right) \\
= & {\left[\partial_{t}-a\left(t, A,\left\{\psi_{k}\right\}\right) A+a\left(t, A,\left\{\psi_{k}-\varphi_{k}\right\}\right) A\right] } \\
& \cdot\left[\partial_{t}-b\left(t, A,\left\{\psi_{k}\right\}\right) A+b\left(t, A,\left\{\psi_{k}-\varphi_{k}\right\}\right) A\right] \\
& -c\left(t, A,\left\{\psi_{k}\right\}\right) A+c\left(t, A,\left\{\psi_{k}-\varphi_{k}\right\}\right) A \\
= & \mathrm{P}\left(\left\{\psi_{k}\right\}\right)+a\left(t, A,\left\{\psi_{k}-\varphi_{k}\right\}\right) A\left[\partial_{t}-b\left(t, A,\left\{\psi_{k}\right\}\right) A\right] \\
& +\left(\partial_{t}-a\left(t, A,\left\{\psi_{k}\right\}\right) A+a\left(t, A,\left\{\psi_{k}-\varphi_{k}\right\}\right) A\right) b\left(t, A,\left\{\psi_{k}-\varphi_{k}\right\}\right) A \\
& +c\left(t, A,\left\{\psi_{k}-\varphi_{k}\right\}\right) A \\
= & \mathrm{P}\left(\left\{\psi_{k}\right\}\right)+R .
\end{aligned}
$$

Now to apply Theorem 2.1 with $\mathbf{P}\left(\left\{\varphi_{k}\right\}\right)$ and $\mathbf{P}\left(\left\{\psi_{k}\right\}\right)$ instead of $\mathbf{P}_{1}$ and $\mathbf{P}_{2}$ or vice versa, we have to prove that $R$ verifies property (2.12), and for this it is sufficient to prove that $a\left(t, A,\left\{\psi_{k}-\varphi_{k}\right\}\right), b\left(t, A,\left\{\psi_{k}-\varphi_{k}\right\}\right), c\left(t, A,\left\{\psi_{k}-\varphi_{k}\right\}\right)$ verify property (2.12).

Let us take, for instance,

$$
a\left(t, A,\left\{\chi_{k}\right\}\right)=\sum_{1}^{\infty} a_{k}(t) \chi_{k}(A) A^{-k},
$$

where we have put $\chi_{k}=\psi_{k}-\varphi_{k}$.

Now it is sufficient to prove that there is an $\epsilon>0$ such that for every $s \in \mathbf{R}, a\left(t, A,\left\{\chi_{k}\right\}\right)$ is a holomorphic function on $U$ with values in the Banach space $L\left(E_{s}, E_{s+\epsilon}\right)$ of the bounded linear operators of $E_{s}$ into $E_{s+\epsilon}$ and because for any given $u$ for which $e^{s A} u$ is defined,

$$
a\left(t, A,\left\{\chi_{k}\right\}\right) e^{s A} u=e^{s A} a\left(t, A,\left\{\chi_{k}\right\}\right) u,
$$

it is enough to consider the case $s=0$.

Because, by hypothesis, $a\left(t, A,\left\{\chi_{k}\right\}\right)$ depends holomorphically on $t$ when $t$ varies in $U$, we have to prove that there is an $\epsilon>0$, independent on $t$, such that for every $u \in H$, the series

$$
\sum_{0}^{\infty} \frac{\epsilon^{h}}{h !} A^{h} a\left(t, A,\left\{\chi_{k}\right\}\right) u
$$

converges in $H$, i.e. that there is a constant $M$, independent of $t$ and $u$, such that

$$
\left\|\sum_{0}^{\infty} \frac{\epsilon^{h}}{h !} A^{h} a\left(t, A,\left\{\chi_{k}\right\}\right) u\right\|_{0} \leqslant M\|u\|_{0} .
$$

We need the following

LEMma 2.1. There is a constant $M_{1}$, independent on $t \in U$, such that 


$$
\left\|A^{h} a\left(t, A,\left\{\chi_{k}\right\}\right) u\right\|_{0} \leqslant M_{1}^{h+1} h !\|u\|_{0}
$$

for every nonnegative integer $h$ and any $u \in H$.

Proof. First of all we observe that $\chi_{k}(\lambda)$, for $\lambda \in \mathbf{R}$, is a real function, such that, $\forall k \geqslant 1$

$$
0 \leqslant \chi_{k}(\lambda) \leqslant 1 \text { and } \operatorname{supp} \chi_{k} \subseteq\left[c_{1} k, c_{2} k+1\right]
$$

if we suppose $c_{1} \leqslant c_{2}$.

Then by (2.4),

$$
\begin{aligned}
& \left\|A^{h} a\left(t, A,\left\{\chi_{k}\right\}\right) u\right\|_{0}=\sum_{1}^{h-1} \sup _{U}\left|a_{k}(t)\right|\left\|A^{h-k} \chi_{k}(A) u\right\|_{0} \\
& +\sum_{0}^{\infty} \sup _{U}\left|a_{h+k}(t)\right|\left\|A^{-k} \chi_{k+h}(A) u\right\|_{0} \\
& \leqslant \sum_{1}^{h-1} c^{k+1} k !\left(2 c_{2} k\right)^{h-k}\|u\|_{0}+\sum_{0}^{\infty} c^{k+h+1}(k+h) ! c_{1}^{-k}(h+k)^{-k}\|u\|_{0} \\
& \leqslant\left\{\left(2 c_{2} h\right)^{h} \sum_{0}^{\infty}\left(\frac{c}{c_{2}}\right)^{k}+c^{h+1} h ! \sum_{0}^{\infty}\left(\frac{c}{c_{1}}\right)^{k}\right\}\|u\|_{0} ;
\end{aligned}
$$

then if we take $M_{1}$ big enough and use the Stirling formula, we have (2.15).

Now we prove (2.14). For every integer $l$ we have

$$
\begin{gathered}
\left\|\sum_{0}^{l} \frac{\epsilon^{h}}{h !} A^{h} a\left(t, A,\left\{\chi_{k}\right\}\right) u\right\|_{0} \leqslant \sum_{0}^{l} \frac{\epsilon^{h}}{h !}\left\|A^{h} a\left(t, A,\left\{\chi_{k}\right\}\right) u\right\|_{0} \\
\leqslant \sum_{0}^{l} \frac{\epsilon^{h}}{h !} M_{1}^{h+1} h !\|u\|_{0} \leqslant M_{1} \sum_{0}^{l}{ }_{h}\left(\epsilon M_{1}\right)^{h}\|u\|_{0} .
\end{gathered}
$$

Then if $\epsilon<1 / M_{1}$, we have (2.14), with

$$
M=M_{1} \sum_{0}^{\infty}{ }_{h}\left(\epsilon M_{1}\right)^{h}
$$

which ends the proof of Theorem 2.2 .

3. Concatenations. Starting from the "formal" operator $P$ given by (2.1) and satisfying the hypotheses (2.1), (2.2) we are going to construct a sequence of formal operators $\mathrm{P}^{0}, \mathrm{P}^{1}, \ldots, \mathrm{P}^{J}, \ldots$ whose coefficients belong to $2{ }_{A}((J))$, still satisfying the properties (2.1). For this construction, we refer to [1].

The first step is to find an operator $\psi(t, A)=\Sigma_{k} \psi_{k}(t) A^{-k} \in Q_{A}((J))$, and a "coefficient" $c^{0}(A)=\Sigma_{k} c_{k}^{0} A^{-k} \in C\left[\left[A^{-1}\right]\right]$, such that the operator 


$$
\mathbf{P}^{0}=(X+\psi(t, A))(Y-\psi(t, A))-c^{0}(A) A
$$

is formally equal to $P$.

Lemma II.4.1 in [1] shows how to construct $\psi(t, A)$ and $c^{0}(A)$.

Let us now put

$$
\begin{aligned}
X^{0} & =\partial_{t}-a^{0}(t, A) A, \quad Y^{0}=\partial_{t}-b^{0}(t, A) A, \\
a^{0}(t, A) & =a(t, A)-\psi(t, A) A^{-1}=\sum_{k} a_{k}^{0}(t) A^{-k}, \\
b^{0}(t, A) & =b(t, A)+\psi(t, A) A^{-1}=\sum_{k} b_{k}^{0}(t) A^{-k}
\end{aligned}
$$

Then

$$
\mathrm{P}^{0}=X^{0} Y^{0}-c^{0}(A) A .
$$

Remark 3.1. The important fact is that the "principal symbol" of $P$ is the same as that of $\mathrm{P}^{0}$; in particular the leading coefficients in the power series $a(t, A)$ and $a^{0}(t, A)$ (respectively $b(t, A)$ and $\left.b^{0}(t, A)\right)$ are the same.

REMARK 3.2. If we suppose that property (2.4) is verified, then from the proof of Lemma II.4.1 in [1] it follows easily, after a shrinking of $J$, that there is a neighborhood $V$ of 0 in $C$ such that $\mathrm{R} \cap V=J$, on which $\psi_{k}(t)$ can be extended as a holomorphic function for every nonnegative integer $k$, we can suppose $V=U$.

Furthermore there is a constant $c_{0}>0$, such that

$$
\left\{\left|a_{k}^{0}(t)\right|,\left|b_{k}^{0}(t)\right|,\left|c_{k}^{0}\right|\right\} \leqslant c_{0}^{k+1} k !, \quad \forall k \in \mathrm{N} \text { and } \forall t \in U .
$$

By property (2.4), to prove (3.3) it is sufficient to see that there is a constant $M$ and $D>0$, such that

$$
\left\{\left|\psi_{k}(t)\right|,\left|c_{k}^{0}\right|\right\} \leqslant D M^{k} k !
$$

$\forall k \in \mathbf{N}, \forall t \in U$ (possibly after a shrinking of $U$ ).

From (II.4.3) of [1] we have that $\psi_{k}(t)$ and $c_{k}^{0}$ have to verify the following equality

$$
\sum_{h=0}^{k} \delta_{h}(t) \psi_{k-h}(t)+c_{k}(t)-c_{k}^{0}=\sum_{h=0}^{k-1} \psi_{h}(t) \psi_{k-1-h}(t)+\psi_{k-1}^{\prime}(t)
$$

where $\delta_{h}(t)=a_{h}(t)-b_{h}(t)$.

We reason by induction. We choose $D$ so big that (3.4) is verified for $k=$ 0 , we suppose (3.4) true up to $k-1$ and we want to prove it for $k$.

Because $\delta_{0}(0)=a_{0}(0)-b_{0}(0)=0,(3.5)$, computed for $t=0$, gives us 


$$
c_{k}^{0}=c_{k}(0)+\sum_{h=1}^{k} \delta_{h}(0) \psi_{k-h}(0)-\sum_{h=1}^{k-1} \psi_{h}(0) \psi_{k-1-h}(0)+\psi_{k-1}^{\prime}(0)
$$

By (2.4), there is a constant $c_{1}$ such that $\left|\delta_{k}(t)\right|,\left|c_{k}(t)\right| \leqslant D c_{1}^{k} k$ ! if we suppose $D>2 c$. Then from (3.6) we have

$$
\begin{aligned}
\left|c_{k}^{0}\right|= & \sum_{h=1}^{k} D c_{1}^{h} h ! \cdot D M^{k-h}(k-h) !+D c_{1}^{k} k ! \\
& +\sum_{1}^{k-1} D^{2} M^{k-1} h !(k-h-1) !+\frac{D M^{k-1}}{d}(k-1) !
\end{aligned}
$$

Indeed, by Cauchy's inequality, if we shrink enough $U$, we have $\left|\psi_{k-1}^{\prime}(t)\right| \leqslant$ $\left(D M^{k-1} / d\right)(k-1) !, \forall t \in U$, where $d$ is a positive constant independent on $k$.

Then if $\alpha$ is a constant such that $0<\alpha \leqslant 1$,

$$
\begin{aligned}
\left|c_{k}^{0}\right| \leqslant & \frac{\alpha}{6} D M^{k} k ! \sum_{1}^{k}\left(\frac{6 D c_{1}}{\alpha M}\right)^{n}+\frac{\alpha}{6} D\left(\frac{6}{\alpha} c_{1}\right)^{k} k ! \\
& +\frac{\alpha}{6} D M^{k} k !\left(\frac{6 D}{\alpha M} \sum_{1}^{k-1} \frac{h !(k-1-h) !}{(k-1) !} \frac{1}{k}+\frac{6}{\alpha d k M}\right) .
\end{aligned}
$$

If we choose $M$ so big that $M \geqslant 12 D c_{1} / \alpha$ and $M>(6 D / \alpha+6 / d \alpha)$ the series $\Sigma_{1}^{\infty}\left(6 D c_{1} / \alpha M\right)^{h}$ converges. We have

$$
\left|c_{k}^{0}\right| \leqslant 1 / 2 \alpha D M^{k} k ! \leqslant D M^{k} k !
$$

which is the second inequality of (3.4).

From (3.5) we get

$$
\begin{aligned}
h_{k}(t) & =\delta_{0}(t) \psi_{k}(t) \\
& =c_{k}^{0}-c_{k}(t)-\sum_{1}^{k} \delta_{h}(t) \psi_{k-h}(t)+\sum_{1}^{k-1}{ }_{h} \psi_{h}(t) \psi_{k-1-h}(t)+\psi_{k-1}^{\prime}(t)
\end{aligned}
$$

for every $t \in U$.

As before, and from (3.7), we have for $\forall t \in U$

$$
\left|h_{k}(t)\right| \leqslant 1 / 2 \alpha D M^{k} k !+1 / 2 \alpha D M^{k} k ! \leqslant \alpha D M^{k} k !
$$

But $h_{k}(0)=0$ and $\delta_{0}(0)=0$; then

$$
h_{k}(t)=\int_{0}^{1} h_{k}^{\prime}(\tau t) d \tau \cdot t, \quad \delta_{0}(t)=\int_{0}^{1} \delta_{0}^{\prime}(\tau t) d \tau \cdot t .
$$

By the Cauchy inequality and from (3.8), possibly after a shrinking of $U$, there is a positive constant $d$ such that 


$$
\left|h_{k}^{\prime}(t)\right| \leqslant \alpha(D / d) M^{k} k ! \quad \forall k \in N .
$$

Now, since $\delta_{0}^{\prime}(0) \neq 0$, once more after shrinking $U$, we can suppose $\delta_{0}^{\prime}(t) \neq$ $0, \forall t \in U$, then

$$
\psi_{h}(t)=\int_{0}^{1} h_{k}^{\prime}(\tau t) d \tau / \int_{0}^{1} \delta_{0}^{\prime}(\tau t) d \tau, \quad t \in U .
$$

Let $D_{1}$ be equal to $\left|\int_{0}^{1} \delta_{0}^{\prime}(\tau t) d \tau\right|$, therefore

$$
\left|\psi_{k}(t)\right| \leqslant\left(\alpha / d D_{1}\right) D M^{k} k !, \quad \forall t \in U .
$$

Now if $d D_{1}$ is $\leqslant 1$ we put $\alpha=d D_{1}$, otherwise we have $\alpha / d D_{1} \leqslant 1$. In any case we get the first inequality of (3.4) which proves the result.

The operator $\mathbf{P}^{0}$ will be the first element in our sequence attached to $\mathbf{P}$. Suppose we have constructed the $j$ th element $\mathrm{P}^{j}=X^{j} Y^{j}-c^{j}(A) A$ we define

$$
\begin{aligned}
\mathbf{P}^{j+1} & =Y^{j} X^{j}-c^{j}(A) A=X^{j} Y^{j}-\left[X^{j}, Y^{j}\right]-c^{j}(A) A \\
& =X^{j} Y^{j}-\left\{c^{j}(A)+\delta_{t}^{j}(t, A)\right\} A
\end{aligned}
$$

where we put $\delta^{j}(t, A)=a^{j}(t, A)-b^{j}(t, A), j=0,1, \ldots$.

Then, as before, taking $\mathbf{P}^{j+1}$ instead of $\mathbf{P}$, we can write $\mathbf{P}^{j+1}$ in the way

$$
P^{j+1}=X^{j+1} Y^{j+1}-c^{j+1}(A) A
$$

and we will have

$$
\begin{aligned}
X^{j+1}= & \partial_{t}-a^{j+1}(t, A) A, \quad Y^{j+1}=\partial_{t}-b^{j+1}(t, A) A \\
& a^{j+1}(t, A)=a(t, A)-\psi^{j+1}(t, A) A^{-1} \\
& b^{j+1}(t, A)=b(t, A)+\psi^{j+1}(t, A) A^{-1}
\end{aligned}
$$

where $\psi^{j}(t, A)=\Sigma_{k} \psi_{k}^{j}(t) A^{-k} \in Q_{A}((J)), c^{j}(A)=\Sigma_{k} c_{k}^{j} A^{-k} \in C\left[\left[A^{-1}\right]\right]$.

REMARK 3.3. $\mathbf{P}^{j+1}$ will have the same "principal symbol" as $\mathbf{P}$, and therefore verifies (2.2) and (2.3). By Remark 3.2 , like $\mathbf{P}^{0}$ it will verify a property analogous to (3.3), possibly after a shrinking of $U$, with $a_{k}^{0}(t), b_{k}^{0}(t), c_{k}^{0}$ and $c_{0}$ by $a_{k}^{j+1}(t), b_{k}^{j+1}(t), c_{k}^{j+1}, c_{j+1}$.

We will suppose that the constant $c_{j}$ has the property

$$
c_{j+1}>c_{j}>c, \quad j \geqslant 0,
$$

where $c$ is the constant (2.4).

From (3.9) follows immediately the

Proposition 3.1. We have, for $j=0,1, \ldots$,

$$
Y^{j} \mathbf{P}^{j}=\mathbf{P}^{j+1} Y^{j},
$$




$$
X^{j} \mathbf{P}^{j+1}=\mathbf{P}^{j} X^{j} .
$$

DEFinirion 3.1. The sequence of formal operators $\left\{P^{i}\right\}_{j>0}$ will be called the $X Y$-concatenation attached to $P$.

Proposition 3.2. Let $\left\{P^{j}\right\}$ be the XY-concatenation attached to P. Then the leading coefficient $c_{0}^{j}$ of $c^{j}(A)$ is given by

$$
c_{0}^{j}=c_{0}(0)+j \delta_{0}^{\prime}(0)
$$

where $\delta_{0}(t)$ is the leading coefficient in $\delta^{0}(t, A)$.

See Proposition II.4.2 of [1].

THEOREM 3.1. Let us suppose that $\left\{\varphi_{k}\right\} \in\left[\tilde{c}_{0}\right]$ and $\tilde{c}_{0}>2 c_{0}$, where $c_{0}$ is given by (3.3). Then $\mathbf{P}\left(\left\{\varphi_{k}\right\}\right)$ and $\mathbf{P}^{0}\left(\left\{\varphi_{k}\right\}\right)$ are true operators and $P\left(\left\{\varphi_{k}\right\}\right)$ is analytic hypoelliptic at $t=0$ if and only if so is $P^{0}\left(\left\{\varphi_{k}\right\}\right)$.

Proof. The proof is similar to that of Theorem 2.2. We observe that

$$
\begin{aligned}
& A^{-1}\left(\mathrm{P}^{0}\left(\left\{\varphi_{k}\right\}\right)-\mathrm{P}\left(\left\{\varphi_{k}\right\}\right)\right) \\
& =\delta\left(t, A,\left\{\varphi_{k}\right\}\right) \psi\left(t, A,\left\{\varphi_{k}\right\}\right)+c\left(t, A,\left\{\varphi_{k}\right\}\right)-c_{0}\left(A,\left\{\varphi_{k}\right\}\right) \\
& -A^{-1}\left(\psi^{2}+\psi_{t}\right)\left(t, A,\left\{\varphi_{k}\right\}\right) \\
& =\sum_{0}^{\infty}{ }_{k}\left(\sum_{0}^{k} \delta_{h}(t) \psi_{k-h}(t) \varphi_{h}(A) \varphi_{k-h}(A)\right) A^{-k}+\sum_{0}^{\infty}{ }_{k}\left(c_{k}(t)-c_{k}^{0}\right) \varphi_{k}(A) A^{-k} \\
& -\sum_{0}^{\infty} k_{k}\left(\sum_{0}^{k}{ }_{h} \psi_{h}(t) \psi_{k-h} \varphi_{h}(A) \varphi_{k-h}(A)\right) A^{-k-1}-\sum_{0}^{\infty} \psi_{k}^{\prime} \varphi_{k}(A) A^{-k-1} \\
& =\left\{\sum_{0}^{\infty} k\left(\sum_{0}^{k} \delta_{h}(t) \psi_{k-h}(t)\right) \varphi_{k}(A) A^{-k}+\sum_{0}^{\infty}{ }_{k}\left(c_{k}(t)-c_{k}^{0}\right) \varphi_{k}(A) A^{-k}\right. \\
& \left.-\sum_{0}^{\infty}\left[\left(\sum_{0}^{k} \psi_{h}(t) \psi_{k-h}(t)\right)-\psi_{k}^{\prime}(t)\right] \varphi_{k+1}(A) A^{-k-1}\right\} \\
& -\sum_{0}^{\infty} \sum_{0}^{k} \psi_{k-h}(t) \delta_{h}(t)\left(\varphi_{k}-\varphi_{h} \varphi_{k-h}\right)(A) A^{-k} \\
& +\sum_{0}^{\infty} \sum_{0}^{k} \psi_{h}(t) \psi_{k-h}(t)\left(\varphi_{k+1}-\varphi_{h} \varphi_{k-h}\right)(A) A^{-k-1} \\
& +\sum_{0}^{\infty} \psi_{k}^{\prime}(t)\left(\varphi_{k+1}-\varphi_{k}\right)(A) A^{-k-1} \text {. }
\end{aligned}
$$


For how $\mathrm{P}^{0}$ has been formally constructed, we have that the sum in the big brackets is zero, then

$$
\begin{aligned}
A^{-1}\left(\mathrm{P}^{0}\left(\left\{\varphi_{k}\right\}\right)-\mathrm{P}\left(\left\{\varphi_{k}\right\}\right)\right) \\
=-\sum_{0}^{\infty} \sum_{0}^{k} \delta_{h}(t) \psi_{k-h}(t)\left(\varphi_{k}-\varphi_{h} \varphi_{k-h}\right)(A) A^{-k} \\
\quad+\sum_{0}^{\infty} \sum_{0}^{k} \psi_{h}(t) \psi_{k-h}(t)\left(\varphi_{k+1}-\varphi_{h} \varphi_{k-h}\right)(A) A^{-k-1} \\
\quad+\sum_{0}^{\infty} \psi_{k}^{\prime}(t)\left(\varphi_{k+1}-\varphi_{k}\right)(A) A^{-k-1} \\
=\widetilde{R} .
\end{aligned}
$$

Then

$$
\mathrm{P}^{0}\left(\left\{\varphi_{k}\right\}\right)-\mathrm{P}\left(\left\{\varphi_{k}\right\}\right)=A \widetilde{R}=R .
$$

Now to apply Theorem 2.1 , we-need to prove that $R$ or $\widetilde{R}$ verifies property (2.12).

Then as in the proof of Theorem 2.2, we reduce ourselves to prove

Lemma 3.1. There is a constant $M_{1}$ independent on $t \in U$, such that

$$
\left\|A^{h} \widetilde{R} u\right\|_{0} \leqslant M_{1}^{h+1} h !\|u\|_{0}
$$

for every nonnegative integer $h$ and any $u \in H$.

Proof. First of all we observe that: $\forall h, 0 \leqslant h \leqslant k, k \geqslant 1$

$$
\varphi_{h} \varphi_{k-h}(\lambda)= \begin{cases}0, & \lambda \leqslant \tilde{c}_{0} k / 2 \\ 1, & \lambda \leqslant \tilde{c}_{0} k+1\end{cases}
$$

Therefore

$$
\left(\varphi_{k}-\varphi_{h} \varphi_{k-n}\right)(\lambda)= \begin{cases}0, & \lambda \leqslant \tilde{c}_{0} k / 2, \lambda \geqslant \tilde{c}_{0} k+1 \\ \leqslant 1, & \text { otherwise. }\end{cases}
$$

(3.19) $\left(\varphi_{k+1}-\varphi_{h} \varphi_{k-h}\right)(\lambda)= \begin{cases}0, & \lambda \leqslant \tilde{c}_{0} k / 2, \lambda \geqslant \tilde{c}_{0}(k+1)+1, \\ \leqslant 1, & \text { otherwise. }\end{cases}$

$$
\left(\varphi_{k+1}-\varphi_{k}\right)(\lambda)= \begin{cases}0, & \lambda \leqslant \widetilde{c_{0}} k, \lambda \geqslant \tilde{c}_{0}(k+1)+1 \\ \leqslant 1, & \text { otherwise }\end{cases}
$$


Now using the first of (3.19) we prove that $(3.18)$ is verified from the sum

$$
\sum_{0}^{\infty} \sum_{0}^{k} \delta_{l}(t) \psi_{k-l}(t)\left(\varphi_{k}-\varphi_{l} \varphi_{k-l}\right)(A) A^{-k}
$$

We proceed in the same way for the other series in $R$. By (2.4), (3.3) and (3.12), $\forall t \in U$

$$
\begin{aligned}
\| A^{h} \sum_{0}^{\infty}{ }_{k} & \sum_{0}^{k}\left(\delta_{l} \psi_{k-l}\right)(t)\left(\varphi_{k}-\varphi_{l} \varphi_{k-l}\right)(A) A^{-k} u \|_{0} \\
\leqslant & \sum_{1}^{h-1} \sum_{0}^{k}\left|\left(\delta_{l} \psi_{k-l}\right)(t)\right|\left\|\left(\varphi_{k}-\varphi_{l} \varphi_{k-l}\right)(A) A^{h-k} u\right\|_{0} \\
& +\sum_{0}^{\infty} \sum_{0}^{k+h}\left|\left(\delta_{l} \psi_{k+h-l}\right)(t)\right|\left\|\left(\varphi_{k+h}-\varphi_{l} \varphi_{k+h-l}\right)(A) A^{-k} u\right\|_{0} \\
\leqslant & \sum_{1}^{h-1} \sum_{0}^{k} 4 c_{0}^{k+2} l !(k-l) !\left(\widetilde{c}_{0} k+1\right)^{h-k} \cdot\|u\|_{0} \\
& +\sum_{0}^{\infty} \sum_{0}^{k+h} 4 c_{0}^{k+h+2} l !(k+h-l) !\left[\frac{\tilde{c}_{0}}{2}(k+h)\right]^{-k} \cdot\|u\|_{0} \\
\leqslant & 4 c_{0}^{2} \sum_{1}^{h-1} k c_{0}^{k} !\left(2 \widetilde{c}_{0} k\right)^{h-k} \cdot\|u\|_{0} \\
& +4 c_{0}^{2} \sum_{0}^{\infty}{ }_{k}(k+h) c_{0}^{k+h}(k+h) ! \frac{2^{k}}{c_{0}^{k}(k+h)^{-k}} \cdot\|u\|_{0} .
\end{aligned}
$$

Then if $M$ is a constant big enough we have

$$
\leqslant M\left(2 \tilde{c}_{0}\right)^{h} h^{h} \sum_{0}^{\infty}{ }_{k}\left(\frac{c_{0}}{\widetilde{c}_{0}}\right)^{k} \cdot\|u\|_{0}+M c_{0}^{h} h ! \sum_{0}^{\infty}\left(\frac{\left(2 c_{0}\right)}{\widetilde{c}_{0}}\right)^{k} \cdot\|u\|_{0} .
$$

Then because $\tilde{c}_{0}>2 c_{0}$, by Stirling formula, we have the (3.18).

With this lemma, we end the proof as in Theorem 2.2.

Let $j$ be a positive integer and let $\mathbf{P}^{0}, \mathbf{P}^{1}, \ldots, \mathbf{P}^{j}$ be the first $j+1$ operators in the $X Y$-concatenation attached to $\mathbf{P}$.

Let $\tilde{c}_{j}$ be a constant bigger than $2 c_{j}$, where $c_{j}$ is the constant given by (3.12) and $\left\{\rho_{k}\right\} \in\left[\widetilde{c_{j}}\right]$.

Then all $\mathbf{P}^{0}\left(\left\{\rho_{k}\right\}\right), \mathbf{P}^{1}\left(\left\{\rho_{k}\right\}\right), \ldots, \mathbf{P}^{i}\left(\left\{\rho_{k}\right\}\right)$ are true operators.

COROLlaRY 3.1. In the previous hypothesis, if for some integer $h, 0 \leqslant$ $h \leqslant j-1, \mathrm{P}^{h+1}\left(\left\{\rho_{k}\right\}\right)$ is analytic hypoelliptic at $t=0$ and we suppose that the following property is verified: 
there is an open neighborhood $J^{\prime}$ of 0 , relatively compact contained in $J$, such that if $u \in D^{\prime}\left(J^{\prime}, H^{-\infty}\right)$ and

$$
Y^{h}\left(\left\{\rho_{k}\right\}\right) u \in A\left(J^{\prime}, H^{\omega}\right), \quad \mathrm{P}^{h}\left(\left\{\rho_{k}\right\}\right) u \in A\left(J^{\prime}, H^{\omega}\right)
$$

then $u \in A\left(J^{\prime}, H^{\omega}\right)$

then also $\mathrm{P}^{h}\left(\left\{\rho_{k}\right\}\right)$ is analytic hypoelliptic at $t=0$.

Proof. From (3.9), we have

$$
\begin{aligned}
Y^{h} & \left(\left\{\rho_{k}\right\}\right) \mathbf{P}^{h}\left(\left\{\rho_{k}\right\}\right) \\
& =\left[X^{h}\left(\left\{\rho_{k}\right\}\right) Y^{h}\left(\left\{\rho_{k}\right\}\right)-\left\{c^{h}\left(A,\left\{\rho_{k}\right\}\right)+\delta_{t}^{h}\left(t, A,\left\{\rho_{k}\right\}\right)\right\} A\right] Y^{h}\left(\left\{\rho_{k}\right\}\right) \\
& =\widetilde{\mathbf{P}}^{h}\left(\left\{\rho_{k}\right\}\right) Y^{h}\left(\left\{\rho_{k}\right\}\right)
\end{aligned}
$$

where we put $\widetilde{\mathbf{P}}^{h}=X^{h} Y^{h}-\left\{c^{h}(A)+\delta_{t}^{h}(t, A)\right\} A$.

Now if we recall as $\mathbf{P}^{h+1}$ has been constructed, from Theorem 3.1 we have that $\widetilde{\mathbf{P}}^{h}\left(\left\{\rho_{k}\right\}\right)$ is analytic hypoelliptic at $t=0$ if and only if so is $\mathbf{P}^{h+1}\left(\left\{\rho_{k}\right\}\right)$.

Then by hypothesis, there is a neighborhood $J^{\prime \prime}$ of 0 that we can suppose equal to $J^{\prime}$, on which $\widetilde{\mathbf{P}}^{h}\left(\left\{\rho_{k}\right\}\right)$ is hypoelliptic.

Then let $u \in D^{\prime}\left(J^{\prime}, H^{-\infty}\right)$ such that $\mathbf{P}^{h}\left(\left\{\rho_{k}\right\}\right) u \in \mathrm{A}\left(J^{\prime}, H^{\omega}\right)$, then

$$
\widetilde{\mathbf{P}}^{h}\left(\left\{\rho_{k}\right\}\right) Y^{h}\left(\left\{\rho_{k}\right\}\right) u=Y^{h}\left(\left\{\rho_{k}\right\}\right) \mathrm{P}^{h}\left(\left\{\rho_{k}\right\}\right) u \in A\left(J^{\prime}, H^{\omega}\right) ;
$$

therefore $Y^{h}\left(\left\{\rho_{k}\right\}\right) u \in A\left(J^{\prime}, H^{\omega}\right)$ then by (3.21) $u \in A\left(J^{\prime}, H^{\omega}\right)$, therefore $\mathbf{P}^{h}\left(\left\{\rho_{k}\right\}\right)$ is analytic hypoelliptic at $t=0$.

4. Statement of the main theorem. Let $\mathbf{P}$ be the formal operator given by (2.1) and satisfying the hypothesis (2.2), (2.3) and (2.4) and let $c$ be as usual, the constant given by (2.4).

We recall that for any sequence $\left\{\rho_{k}\right\} \in\left[c_{1}\right]$, where $c_{1}>c, \mathbf{P}\left(\left\{\rho_{k}\right\}\right)$ is a true operator.

DEFINITION 4.1. The formal operator $\mathbf{P}$ is said to be analytic hypoelliptic at a point $t \in J$ if for any constant $c_{1} \geqslant c$ and for any sequence $\left\{\rho_{k}\right\} \in\left[c_{1}\right]$, the true operator $\mathrm{P}\left(\left\{\rho_{k}\right\}\right)$ is analytic hypoelliptic at $t$.

REMARK 4.1. By Theorem 2.2, for $\mathbf{P}$ to be analytic hypoelliptic at a point $t \in J$, it is necessary and sufficient that there is a constant $c_{1}$ bigger than $c$ and a sequence $\left\{\rho_{k}\right\} \in\left[c_{1}\right]$ for which $\mathrm{P}\left(\left\{\rho_{k}\right\}\right)$ is analytic hypoelliptic at $t$.

We can now state the main theorem of this paper.

THEOREM 4.1. Let $\mathbf{P}$ be the formal operator given by (2.1) and satisfying the hypotheses (2.2), (2.3) and (2.4); let $\mathbf{P}^{j}=X^{j} Y^{j}-c^{j}(A) A, j=0,1, \ldots$, denote the successive elements in the $X Y$-concatenation attached to $\mathbf{P}$.

The following are equivalent:

(4.1) P is hypoelliptic at $t=0$; 
(4.2) $\mathrm{P}$ is analytic hypoelliptic at $t=0$;

(4.3) For no integer $j \geqslant 0$, the formal power series in $A^{-1}, c^{j}(A)$, is identically zero.

The equivalences $(4.1) \Leftrightarrow(4.3)$ have been already proved in [1]. We have to prove that (4.3) implies (4.2) (by Definition 1.2, (4.2) $\Rightarrow(4.1)$ ).

For the proof of Theorem 4.1 we need the following theorem which will be proved in $\S 6$.

THEOREM 4.2. Let I be an open interval centered in the origin and $\bar{I}$ its closure. Suppose that $Q$ is a (true) operator given by

$$
Q=\left(\partial_{t}-\alpha(t) t A-\widetilde{\alpha}(t, A)\right)\left(\partial_{t}-\beta(t) t A-\widetilde{\beta}(t, A)\right)-\gamma(t) A-\widetilde{\gamma}(t, A)
$$

where $\alpha(t), \beta(t), \gamma(t)$ are complex valued $C^{\infty}$ functions on $\bar{I}$ and $\widetilde{\alpha}(t, A), \widetilde{\beta}(t, A)$, $\tilde{\gamma}(t, A)$ are $C^{\infty}$ functions on $\bar{I}$ with values in $L(H, H)$, for which the following hypotheses are verified:

$$
\begin{gathered}
\operatorname{Re} \alpha(0)>0, \quad \operatorname{Re} \beta(0)<0 ; \\
|\alpha(0)-\beta(0)|^{2} \leqslant 2 \operatorname{Re}\{\gamma(0)[\overline{\alpha(0)-\beta(0)}]\} .
\end{gathered}
$$

Suppose furthermore that there is a constant $M>0$, such that for every $t \in I$ and any nonnegative integer $h$

$$
\begin{aligned}
& \left\{\left|\partial_{t}^{h} \alpha(t)\right|,\left|\partial_{t}^{h} \beta(t)\right|,\left|\partial_{t}^{h} \gamma(t)\right|,\left\|\partial_{t}^{h} \widetilde{\alpha}(t, A)\right\|,\right. \\
& \left.\left\|\partial_{t}^{h} \widetilde{\beta}(t, A)\right\|,\left\|\partial_{t}^{h} \widetilde{\gamma}(t, A)\right\|\right\} \leqslant M^{h+1} h !
\end{aligned}
$$

where \|\| is the norm in $L(H, H)$.

Under these hypotheses $\mathbf{P}$ is analytic hypoelliptic at $t=0$.

5. Proof of Theorem 4.1.

Proof of (4.3) $\Rightarrow(4.2)$. Let $P$ be the operator given in Theorem 4.1 and $j$ and integer such that

$$
j \geqslant 1 / 2-\operatorname{Re}\left(c(0) / \delta_{0}^{\prime}(0)\right) .
$$

Let $\tilde{c}_{j}$ be a constant larger than $2 c_{j}$, where $c_{j}$ is given by (3.12), and $\left\{\rho_{k}\right\}$ a sequence belonging to $\left[\tilde{c}_{j}\right]$; then $\mathbf{P}\left(\left\{\rho_{k}\right\}\right), \mathbf{P}^{0}\left(\left\{\rho_{k}\right\}\right), \ldots, \mathbf{P}^{j}\left(\left\{\rho_{k}\right\}\right)$ are true operators.

First of all we observe that $\mathbf{P}^{j}\left(\left\{\rho_{k}\right\}\right)$ verifies the hypotheses of Theorem 4.2.

Because the leading coefficients of $a^{j}(t, A), b^{j}(t, A)$ are the same as those of $a(t, A), b(t, A)$ by (2.2) and (2.3), we have that $\mathrm{P}^{j}\left(\left\{\rho_{k}\right\}\right)$ can be written in the form (4.4); and (4.5) will be verified.

Furthermore by (3.15) and (5.1), since $\gamma(0)=c_{0}^{j},(4.6)$ is verified; finally by Remark 3.3 and by Cauchy's inequality used in the same way as in Remark 
2.1, we see that also (4.7) is verified, therefore we may conclude that $\mathrm{P}^{j}\left(\left\{\rho_{k}\right\}\right)$ is analytic hypoelliptic at $t=0$.

Now we want to prove that under the hypothesis (4.3), starting from the analytic hypoellipticity at $t=0$ of $\mathrm{P}^{j}\left(\left\{\rho_{k}\right\}\right)$, all the $\mathrm{P}^{h}\left(\left\{\rho_{k}\right\}\right), h=0,1, \ldots$, $j-1$, are analytic hypoelliptic at $t=0$, which will imply by Theorem 3.1 that $\mathrm{P}\left(\left\{\rho_{k}\right\}\right)$ is analytic hypoelliptic at $t=0$. By Remark 4.1 this will prove Theorem 4.1. We shall use Corollary 3.1 , and we will have to prove that (3.21) is verified.

We will do the first step; for the others we proceed in the same way.

LEMma 5.1. Under the previous hypotheses (in particular, that $\mathrm{P}^{j}\left(\left\{\rho_{k}\right\}\right)$ is hypoelliptic analytic in $J)$, there is an open neighborhood $J^{\prime}$ of 0 , relatively compact, contained in $J$, such that if $u \in D^{\prime}\left(J^{\prime}, H^{-\infty}\right)$ and

$$
Y^{j-1}\left(\left\{\rho_{k}\right\}\right) u \in A\left(J^{\prime}, H^{\omega}\right), \quad P^{j-1}\left(\left\{\rho_{k}\right\}\right) u \in A\left(J^{\prime}, H^{\omega}\right),
$$

then $u \in A\left(J^{\prime}, H^{\omega}\right)$.

Proof. We set $Y^{j-1}\left(\left\{\rho_{k}\right\}\right) u=g, \mathrm{P}^{j-1}\left(\left\{\rho_{k}\right\}\right) u=h$; then by (5.2), $g$ and $h \in A\left(J^{\prime}, H^{\omega}\right)$; therefore

$$
c^{j-1}\left(A,\left\{\rho_{k}\right\}\right) A u=X^{j-1}\left(\left\{\rho_{k}\right\}\right) g-h \in A\left(J^{\prime}, H^{\omega}\right) .
$$

Let $m_{j-1}$ be the smallest integer such that $c_{m_{j-1}}^{j-1} \neq 0$; such an integer exists, by (4.3).

Therefore we can find a number $\rho>0$, so large that

$$
\rho\left|c_{m_{j-1}}^{j-1}\right|^{2}>\sup _{t \in j}\left|b_{0}(t)\right| \text {. }
$$

Let

$$
\begin{aligned}
\gamma\left(A,\left\{\rho_{k}\right\}\right) & =\overline{c_{m_{j-1}}^{j-1}} \cdot c^{j-1}\left(A,\left\{\rho_{k}\right\}\right) A^{m_{j-1}} \\
& =\left|c_{m_{j-1}^{j-1}}^{j}\right|^{2} \rho_{m_{j-1}}(A)+\sum_{m_{j-1}+1}^{\infty} \overline{c_{m_{j-1}}^{j-1}} c_{k}^{j-1} \rho_{k}(A) A^{m_{j-1}-k}
\end{aligned}
$$

Then

$$
\begin{aligned}
\lambda\left(A,\left\{\rho_{k}\right\}\right) & =\left|c_{m_{j-1}^{j-1}}^{j-1}\right|^{2}+\sum_{m_{j-1}+1}^{\infty} \overline{c_{m_{j-1}}^{j-1}} c_{k}^{j-1} \rho_{k}(A) A^{m_{j-1}-k} \\
& =\gamma\left(A,\left\{\rho_{k}\right\}\right)+\left|c_{m_{j-1}}^{j-1}\right|^{2}\left(1-\rho_{m_{j-1}}(A)\right)=\gamma\left(A,\left\{\rho_{k}\right\}\right)+R,
\end{aligned}
$$

where $R=\left|c_{m_{j-1}}^{j-1}\right|^{2}\left(1-\rho_{m_{j-1}}(A)\right)$.

Now it is easy to see $R$ verifies a property analogous to (3.18), then it will satisfy property (2.12) and by the fact that $\mathrm{P}^{j-1}\left(\left\{\rho_{k}\right\}\right) u \in A\left(J^{\prime}, H^{\omega}\right)$, we will 
get, as in the proof of Theorem 2.1, that $R u \in A\left(J^{\prime}, H^{\omega}\right)$.

Then, by (5.3), $\rho \lambda\left(A,\left\{\rho_{k}\right\}\right) A u \in A\left(J^{\prime}, H^{\omega}\right)$. Therefore

$$
Z^{j-1} u=Y^{j-1}\left(\left\{\rho_{k}\right\}\right) u-\rho \lambda\left(A,\left\{\rho_{k}\right\}\right) A u \in A\left(J^{\prime}, H^{\omega}\right)
$$

and by virtue of the ellipticity of $Z^{j-1}$, due to (5.4), we conclude that $u \in$ $A\left(J^{\prime}, H^{\omega}\right)$, which proves the lemma.

As a consequence, by Corollary 3.1 we can conclude that $\mathrm{P}^{j-1}\left(\left\{\rho_{k}\right\}\right)$ is analytic hypoelliptic at $t=0$.

By repeating these arguments $j-1$ times we reach the conclusion that $\mathbf{P}^{0}\left(\left\{\rho_{k}\right\}\right)$ and (see Theorem 3.1) $\mathbf{P}\left(\left\{\rho_{k}\right\}\right)$ are analytic hypoelliptic at $t=0$.

This ends the proof of Theorem 4.1, by Remark 4.1.

6. Proof of Theorem 4.2. We follow closely the argument in [2]. Let us consider the operator

$$
Q=\left(\partial_{t}-\alpha(t) t A-\widetilde{\alpha}(t, a)\right)\left(\partial_{t}-\beta(t) t A-\widetilde{\beta}(t, A)\right)-\gamma(t) A-\tilde{\gamma}(t, A) .
$$

If we redefine the coefficients appropriately, we can write $Q$ in the form

$$
Q=\partial_{t}^{2}+\alpha(t) t \partial_{t} A+\beta(t) t^{2} A^{2}+\gamma(t, A) \partial_{t}+\lambda(t, A) A+\mu(t, A)
$$

where $\alpha(t), \beta(t)$ are complex valued $C^{\infty}$ functions and $\gamma(t, A), \lambda(t, A), \mu(t, A)$ are $C^{\infty}$ functions, defined in $I$, with values in $L(H, H)$. There is a constant $M>0$, such that, for every $t \in I$,

(6.2) $\left\{\left|\partial_{t}^{h} \alpha(t)\right|,\left|\partial_{t}^{h} \beta(t)\right|,\left\|\partial_{t}^{h} \gamma(t, A)\right\|,\left\|\partial_{t}^{h} \lambda(t, A)\right\|,\left\|\partial_{t}^{h} \mu(t, A)\right\|\right\} \leqslant M^{h+1} h !$

where \|\| is the norm in $L(H, H)$.

Because of the hypotheses (4.5) and (4.6), by Corollary II.2.1 of [1], if we shrink enough $I$, there is a constant $c>0$, such that

$$
\int\left(\left\|\partial_{t} v\right\|_{0}^{2}+\|t A v\|_{0}^{2}\right) d t \leqslant c\left|\int(Q v, v)_{0} d t\right|, \quad \forall v \in C_{c}^{\infty}\left(I, H^{\infty}\right),
$$

where $(,)_{0}$ is the inner product of $H$.

If we use the norm \|\|$_{s, k}$ introduced in [4], the inequality (6.3) can be written

$$
\|v\|_{1,1}^{2} \leqslant c\left|\int(Q v, v)_{0} d t\right|
$$

whence, by Schwarz' inequality,

which implies

$$
\|v\|_{1,1}^{2} \leqslant c\|Q u\|_{-1,-1}\|v\|_{1,1},
$$

Therefore, as shown in [4], (6.3) implies 


$$
\|v\|_{2,2} \leqslant c\|Q v\|_{0,0}=c\left\{\int\|Q v\|_{0}^{2} d t\right\}^{1 / 2},
$$

$\forall v \in C_{c}^{\infty}\left(I, H^{\infty}\right)$. If we use the notation $\|f\|^{2}=\int\|f\|_{0}^{2} d t$, a norm equivalent to \|\|$_{2,2}$ is given by

$$
N(v)^{2}=\left\|\partial_{t}^{2} v\right\|^{2}+\left\|t \partial_{t} A v\right\|^{2}+\left\|t^{2} A^{2} v\right\|^{2}+\left\|\partial_{t} v\right\|^{2}+\|A v\|^{2}+\|v\|^{2} .
$$

When the integration with respect to $t$ is performed over some interval $w$ we write $N_{w}(v)$.

Let us put

$$
\begin{aligned}
\Gamma_{p}= & \left\{(h, k) \in \mathrm{N}^{2} \mid h \leqslant 4, h+k \leqslant p-h\right\} \\
& \cup\left\{(h, k) \in \mathrm{N}^{2} \mid h \geqslant 4, h+k \leqslant p-4\right\} .
\end{aligned}
$$

Note that $\Gamma_{p}$ contains all the pairs $(h, k)$ such that $h+k \leqslant p-4$.

Now let:

$$
N_{\omega}^{p}(v)=\sup _{(h, k) \in \Gamma_{p}} N_{\omega}\left(\partial_{t}^{h} A^{k_{v}}\right)
$$

and let $u \in D^{\prime}\left(I, H^{-\infty}\right)$ be such that

$$
Q u=f \in A\left(I, H^{\omega}\right) \text {. }
$$

We want to prove that there is an open subinterval $J$ of $I$ centered in 0 , independent of $u$, such that

$$
u \in A\left(J, H^{\omega}\right) .
$$

First of all (6.8) implies that

$$
u \in C^{\infty}\left(I, H^{\infty}\right),
$$

since $Q$ is hypoelliptic in a suitable interval $J$ (cf. [1, Corollary II.3.2])

Therefore, in order to prove (6.9) it suffices to show that we can choose $J$ and a constant $B$ such that

$$
N_{j}^{p}(u) \leqslant B^{p+1} p ! \quad \forall p \in N, p \geqslant p_{0},
$$

where $p_{0}$ is a fixed integer. By the standard embedding theorems, (6.11) will imply

$$
\sup _{J}\left\|\partial_{t}^{h} A^{k} u\right\|_{0} \leqslant C B_{1}^{h+k}(h+k) !
$$

where $B$ and $C$ are suitable constants.

Possibly after shrinking $I$, we may suppose that, for some $c>0$,

$$
\sup _{I}\left\|\partial_{t}^{h} A^{k} Q u\right\|_{0} \leqslant C^{h+k+1}(h+k) ! .
$$

Let $\omega=]-2,2$ [, we can always suppose that $\omega$ is contained in $I$ and put $\omega_{\delta}=-2+\delta, 2-\delta[$ with $0 \leqslant \delta<2$. 
Let $\epsilon$ and $\epsilon_{1}$ be two real numbers, $0<\epsilon, \epsilon_{1}<1$, and $\rho(t) \in C_{c}^{\infty}\left(\omega_{\epsilon_{1}}\right)$ such that $\rho(t)=1, \forall t \in \omega_{\epsilon+\epsilon_{1}}$, and $\left|\partial_{t}^{\alpha} \rho\right| \leqslant C_{1} \epsilon^{-\alpha}, 0 \leqslant \alpha \leqslant 2$. By (6.5), if $p \geqslant \alpha$ we have that

$$
\begin{aligned}
& N_{\omega_{\epsilon+\epsilon_{1}}}\left(\partial_{t}^{h} A^{k} u\right) \leqslant N\left(\rho \partial_{t}^{h} A^{k} u\right) \leqslant C\left\|Q \rho \partial_{t}^{h} A^{k} u\right\| \\
& \leqslant C\left\{\left\|\rho \partial_{t}^{h} A^{k} Q u\right\|+\left\|[Q, \rho] \partial_{t}^{h} A^{k} u\right\|+\left\|\rho\left[Q, \partial_{t}^{h} A^{k}\right] u\right\|\right\},
\end{aligned}
$$

$$
\forall(h, k) \in \Gamma_{p}
$$

From now on, $C$ will denote a generic constant independent of $h, k, p, \epsilon$ and $\epsilon_{1}$.

Now, by (6.12), we have

$$
N_{\omega_{\epsilon+\epsilon}}\left(\partial_{t}^{h} A^{k} u\right) \leqslant C\left\{C^{p} p !+\left\|[Q, \rho] \partial_{t}^{h} A^{k} u\right\|+\left\|\rho\left[Q, \partial_{t}^{h} A^{k}\right] u\right\|\right\} .
$$

LEMMA 6.1. Under the preceding hypotheses,

$$
\left\|[Q, \rho] \partial_{t}^{h} A^{k} u\right\| \leqslant C\left\{\epsilon^{-1} N_{\omega_{\epsilon_{1}}}^{p-1}(u)+\epsilon^{-2} N_{\omega_{\epsilon_{1}}}^{p-2}(u)\right\} .
$$

Proof.

$$
\begin{aligned}
\left\|[Q, \rho] \partial_{t}^{h} A^{k} u\right\| \leqslant & 2\left\|\rho^{\prime} \partial_{t} \partial_{t}^{h} A^{k} u\right\|+\left\|\rho^{\prime \prime} \partial_{t}^{h} A^{k} u\right\| \\
& +\left\|\alpha(t) \rho^{\prime} t \partial_{t}^{h} A^{k+1} u\right\|+\left\|\gamma(t, A) \rho^{\prime} \partial_{t}^{h} A^{k} u\right\| .
\end{aligned}
$$

Let us estimate $\left\|\rho^{\prime} \partial_{t} \partial_{t}^{h} A^{k} u\right\|$. Suppose $k \geqslant 1$; since:

$$
\left|\rho^{\prime} / t\right| \leqslant C \epsilon^{-1}
$$

we have

$$
\begin{aligned}
& \left\|\rho^{\prime} \partial_{t} \partial_{t}^{h} A^{k} u\right\| \leqslant C \epsilon^{-1}\left\|t \partial_{t} \partial_{t}^{h} A^{k} u\right\|_{\omega_{\epsilon_{1}}} \\
& \leqslant C \epsilon^{-1}\left\|t \partial_{t} A\left(\partial_{t}^{h} A^{k-1} u\right)\right\|_{\omega_{\epsilon_{1}}} \leqslant C \epsilon^{-1} N_{\omega_{\epsilon_{1}}}\left(\partial_{t}^{h} A^{k-1} u\right) .
\end{aligned}
$$

If $h \geqslant 4, h+k \leqslant p-4 \Rightarrow h+k-1 \leqslant p-1-4 \Rightarrow(h, k-1) \in \Gamma_{p-1}$; analogously, if $h \leqslant 4,(h, k-1) \in \Gamma_{p-1}$, then $\left\|\rho^{\prime} \partial_{t} \partial_{t}^{h} A^{k} u\right\| \leqslant C \epsilon^{-1} N_{\omega_{\epsilon_{1}}}^{p-1}(u)$.

Suppose $k=0$. Then we can suppose $h \geqslant 1$ and

$$
\left\|\rho^{\prime} \partial_{t} \partial_{t}^{h} u\right\| \leqslant\left\|\rho^{\prime} \partial_{t} \partial_{t}^{h-1} u\right\| \leqslant C \epsilon^{-1} N_{\omega_{\epsilon_{1}}}\left(\partial_{t}^{h-1} u\right) \leqslant C \epsilon^{-1} N_{\omega_{\epsilon_{1}}}^{p-1}(u),
$$

because, as before, we have $(h-1,0) \in \Gamma_{p-1}$. Then in general we have $\left\|\rho^{\prime} \partial_{t} \partial_{t}^{h} A^{k} u\right\| \leqslant C \epsilon^{-1} N_{\omega_{\epsilon_{1}}}^{p-1}(u)$.

Let us estimate $\left\|\alpha(t) \rho^{\prime} t \partial_{t}^{h} A^{k+1} u\right\|$. Suppose $k \geqslant 1$; by (6.13) we have 


$$
\begin{aligned}
\left\|\rho^{\prime} t \partial_{t}^{h} A^{k+1} u\right\| & \leqslant C \epsilon^{-1}\left\|t^{2} A^{2}\left(\partial_{t}^{h} A^{k+1} u\right)\right\|_{\omega_{\epsilon_{1}}} \\
& \leqslant C \epsilon^{-1} N_{\omega_{\epsilon_{1}}}\left(\partial_{t}^{h} A^{k-1} u\right) \leqslant C \epsilon^{-1} N_{\omega_{\epsilon_{1}}^{p-1}}(u) .
\end{aligned}
$$

If $k=0$ we can suppose $h \geqslant 1$; then

$$
\begin{aligned}
\left\|\rho^{\prime} t \partial_{t}^{h} A u\right\| & \leqslant C \epsilon^{-1} \|_{t \partial_{t} A\left(\partial_{t}^{h-1} u\right) \|_{\omega_{\epsilon_{1}}}} \\
& \leqslant C \epsilon^{-1} N_{\omega_{\epsilon_{1}}}\left(\partial_{t}^{h-1} u\right) \leqslant C \epsilon^{-1} N_{\omega_{\epsilon_{1}}^{p-1}}(u) .
\end{aligned}
$$

Then in general $\left\|\alpha(t) \rho^{\prime} t \partial_{t}^{h} A^{k+1} u\right\| \leqslant C \epsilon^{-1} N_{\omega_{\epsilon}}^{p-1}(u)$.

If we consider $\left\|\rho^{\prime \prime} \partial_{t}^{h} A^{k} u\right\|,\left\|\gamma(t, A) \rho^{\prime} \partial_{t}^{h} A^{k} u\right\|$, we have that both of them are $\leqslant C \epsilon^{-2}\left\|\partial_{t}^{h} A^{k} u\right\|_{\omega_{\epsilon_{1}}}$.

Now if $h \geqslant 2$,

$$
\left\|\partial_{t}^{h} A^{k} u\right\|_{\omega_{\epsilon_{1}}}=\left\|\partial_{t}^{2}\left(\partial_{t}^{h-2} A^{k} u\right)\right\|_{\omega_{\epsilon_{1}}} \leqslant N_{\omega_{\epsilon_{1}}}\left(\partial_{t}^{h-2} A^{k} u\right) \leqslant N_{\omega_{\epsilon_{1}}}^{p-2}(u)
$$

if $h=1$,

$$
\left\|\partial_{t} A^{k} u\right\|_{\omega_{\epsilon_{1}}} \leqslant N_{\omega_{\epsilon_{1}}}\left(A^{k} u\right) \leqslant N_{\omega_{\epsilon_{1}}^{p-2}(u)}^{p}
$$

because $1+k \leqslant p-1 \Rightarrow k \leqslant p-2 \Rightarrow(0, k) \in \Gamma_{p-2}$.

If $h=0$, we can suppose $k \geqslant 2$; then, since $\left|\rho^{\prime \prime}\right| t^{2} \mid \leqslant c \epsilon^{-2}$, and by (6.13),

$$
\begin{aligned}
\left\|\rho^{\prime \prime} A^{k} u\right\|,\left\|\gamma(t, A) \rho^{\prime} A^{k} u\right\| & \leqslant C \epsilon^{-2}\left\|t^{2} A^{k} u\right\|_{\omega_{\epsilon_{1}}} \\
& \leqslant c \epsilon^{-2} N_{\omega_{\epsilon_{1}}}\left(A^{k-2} u\right) \leqslant c \epsilon^{-2} N_{\omega_{\epsilon_{1}}^{p-2}(u)}
\end{aligned}
$$

then in general

$$
\left\|\rho^{\prime \prime} \partial_{t}^{h} A^{k} u\right\|,\left\|\gamma(t, A) \rho^{\prime} \partial_{t}^{h} A^{k} u\right\| \leqslant c \epsilon^{-2} N_{\omega_{\epsilon_{1}}}^{p-2}(u),
$$

which proves the lemma.

LEMMA 6.2. Same hypotheses as in Lemma 6.1.

$$
\left\|\rho\left[Q, \partial_{t}^{h} A^{k}\right] u\right\| \leqslant C \sum_{k=1}^{p} C^{k} \frac{p !}{(p-k) !} N_{\omega_{\epsilon_{1}}}^{p-k}(u) .
$$

Proof.

$$
\begin{aligned}
&\left\|\rho\left[Q, \partial_{t}^{h} A^{k}\right] u\right\|=\left\|\rho\left[Q, \partial_{t}^{h}\right] A^{k} u\right\| \\
& \leqslant\left\|\rho\left[\alpha(t) t, \partial_{t}^{h}\right] \partial_{t} A^{k+1} u\right\| \\
&+\left\|\rho\left[\beta(t) t^{2} \partial_{t}^{h}\right] A^{k+2} u\right\|+\left\|\rho\left[\gamma(t, A), \partial_{t}^{h}\right] \partial_{t} A^{k} u\right\| \\
&+\left\|\rho\left[\lambda(t, A), \partial_{t}^{h}\right] A^{k+1} u\right\|+\left\|\rho\left[\mu(t, A), \partial_{t}^{h}\right] A^{k} u\right\| .
\end{aligned}
$$


We may suppose $h \geqslant 1$. We have, first,

$$
\begin{aligned}
\left\|\rho\left[\alpha(t) t, \partial_{t}^{h}\right] \partial_{t} A^{k+1} u\right\| & \\
\leqslant & \sum_{\lambda+\nu=h ; \nu<\alpha} \frac{h !}{\lambda ! \nu !}\left\|\left(\partial_{t}^{\lambda} \alpha\right) \rho t \partial_{t} A\left(\partial_{t}^{\nu} A^{k} u\right)\right\| \\
& +\sum_{\lambda+\nu+1=h} \frac{h !}{\lambda ! \nu !}\left\|\left(\partial_{t}^{\lambda} \alpha\right) \rho\left(\partial_{t}^{\nu+1} A^{k+1} u\right)\right\| .
\end{aligned}
$$

By (6.2), it suffices to prove that

$$
\begin{aligned}
& \| \rho t \partial_{t} A\left(\partial_{t} A^{k} u \| \leqslant C N_{\omega_{\epsilon_{1}}}^{p-\lambda}(u),\right. \\
& \left\|\rho \partial_{t}^{\nu+1} A^{k+1} u\right\| \leqslant C N_{\omega_{\epsilon_{1}}-\lambda-1}^{p-\lambda}(u) .
\end{aligned}
$$

Suppose $h \geqslant 4$. For the first inequality (6.16), with $(h, k) \in \Gamma_{p}$, we have $\lambda+\nu+k \leqslant p-4 \Rightarrow p-\lambda-4 \Rightarrow(\nu, k) \in \Gamma_{p-\lambda}$. Consequently,

$$
\left\|\rho t \partial_{t} A\left(\partial_{t}^{\nu} A^{k} u\right)\right\| \leqslant C N_{\omega_{\epsilon_{1}}}\left(\partial_{t}^{\nu} A^{k} u\right) \leqslant C N_{\omega_{\epsilon_{1}}-\lambda}^{p-\lambda}(u) .
$$

As for the second inequality (6.16) we have (1) if $\nu \geqslant 1, \lambda+\nu+1+k \leqslant p-$ $4 \Rightarrow \nu-1+k+1 \leqslant(p-\lambda-1)-4 \Rightarrow(\nu-1, k+1) \in \Gamma_{p-\lambda-1}$, and therefore

$$
\left\|\rho\left(\partial_{t}^{\nu+1} A^{k+1} u\right)\right\| \leqslant C N_{\omega_{\epsilon_{1}}}\left(\partial_{t}^{\nu-1} A^{k+1} u\right) \leqslant C N_{\omega_{\epsilon_{1}}-\lambda-1}^{p-\lambda}(u)
$$

(2) if $\nu=0, \lambda+1+k \leqslant p-4 \Rightarrow 1+k \leqslant p-\lambda-4 \leqslant(p-\lambda-1)-4 \Rightarrow$ $(0, k+1) \in \Gamma_{p-\lambda-1}$, and

$$
\left\|\rho \partial_{t} A^{k+1} u\right\| \leqslant C N_{\omega_{\epsilon_{1}}}\left(A^{k+1} u\right) \leqslant C N_{\omega_{\epsilon_{1}}}^{p-\lambda-1}(u) .
$$

Similar argument when $h \leqslant 4$. As a consequence of $(6.16)$ we see that $\left\|\rho\left[\alpha(t) t, \partial_{t}^{h}\right] \partial_{t} A u\right\|$ verifies the estimate (6.14).

We operate in the same way with $\left\|\rho\left[\beta(t) t^{2}, \partial_{t}^{h}\right] A^{k+2} u\right\|_{\text {. }}$

Next we see, by (6.2), that

$$
\begin{aligned}
\left\|\rho\left[\gamma(t, A), \partial_{t}^{h}\right] \partial_{t} A^{k} u\right\| \leqslant \sum_{\nu<h}\left(\begin{array}{l}
h \\
\nu
\end{array}\right) \rho_{t} \partial_{t}^{h-\nu} \gamma(t, A) \cdot \partial_{t}^{\nu+1} A^{k} u \| \\
\leqslant \sum_{\nu<h}\left(\begin{array}{l}
h \\
\nu
\end{array}\right) c^{h-\nu+1}(h-\nu) !\left\|\rho \partial_{t}\left(\partial_{t}^{\nu} A^{k} u\right)\right\| \\
\leqslant \sum_{\nu<h} c^{h-\nu+1} \frac{h !}{\nu !} N_{\omega_{\epsilon_{1}}}\left(\partial_{t}^{\nu} A^{k} u\right)
\end{aligned}
$$

But if $h \geqslant 4, h+k \leqslant p-4 \Rightarrow \nu+k \leqslant p-(h-\nu)-4 \Rightarrow(\nu, k) \in \Gamma_{p-(h-\nu)}$. If $h \leqslant 4, h+k \leqslant p-h \Rightarrow \nu+k \leqslant p-(h-\nu)-h \leqslant p-(h-\nu)-\nu \Rightarrow$ 
$(\nu, k) \in \Gamma_{p-(h-v)}$. Therefore

$$
\left\|\rho\left[\gamma(t, A), \partial_{t}^{h}\right] \partial_{t} A^{k} u\right\| \leqslant \sum_{\nu<h} c^{h-\nu+1} \frac{h !}{\nu !} N_{\omega_{\epsilon_{1}}}^{p-(h-\nu)}(u) .
$$

We obtain a similar estimate for $\left\|\rho\left[\lambda(t, A), \partial_{t}^{h}\right] A^{k-1} u\right\|$ and $\left\|\rho\left[\mu(t, A), \partial_{t}^{h}\right] A^{k} u\right\|$ and the lemma is thus proved.

By Lemmas 6.1 and 6.2, we get that there is a constant $c>0$ such that, $\forall p \in N, p \geqslant 4, \forall(h, k) \in \Gamma_{p}$,

$$
\begin{aligned}
& N_{\omega_{\epsilon+\epsilon_{1}}}\left(\partial_{t}^{h} A^{k} u\right) \leqslant C\left\{C^{p} p !+\sum_{k=1}^{p} C^{k} \frac{p !}{(p-k) !} N_{\omega_{\epsilon_{1}}^{p-k}(u)}\right. \\
& \left.+\sum_{1}^{2} \epsilon^{-k} N_{\omega_{\epsilon_{1}}^{p-k}(u)}^{p}\right\}
\end{aligned}
$$

whence:

$$
\begin{aligned}
N_{\omega_{\epsilon+\epsilon_{1}}}^{p}(u) \leqslant C\left\{C^{p} p !+\sum_{k=1}^{p} C^{k} \frac{\epsilon^{k} p !}{(p-k) !} \epsilon^{-k} N_{\omega_{\epsilon_{1}}^{p-k}(u)}^{p}\right. & +\sum_{k=1}^{2} \epsilon^{-k} N_{\left.\omega_{\epsilon_{1}}^{p-k}(u)\right\}}
\end{aligned}
$$

Let $l$ be an integer such that $l \geqslant p+4, \epsilon=1 / l, \epsilon_{1}=p / l$ and put

$$
d_{p}=N_{\omega_{(p+1) / l}^{p}}(u) l^{-p-4} .
$$

The inequality (6.17) can be rewritten

$$
d_{p} \leqslant C\left\{C^{p}+\sum_{k=1}^{p} C^{k} d_{p-k}+\sum_{k=1}^{2} d_{p-k}\right\}
$$

for every integer $p \geqslant 4, p \leqslant l-4$, which easily implies, for some $B>0$,

$$
d_{p} \leqslant B^{p+1} \quad \forall p \leqslant l-4
$$

In turn (6.18) implies, for all $l \geqslant 4$,

$$
N_{\omega_{1}}^{l-4}(u) \leqslant B^{l-3} l^{l}
$$

Finally, after an increase of the constant $B$ we get (6.11) with $J=\omega_{1}$.

\section{REFERENCES}

1. F. Trèves, Concatenations of second order evolution equation applied to local solvability and hypoellipticity, Comm. Pure Appl. Math. 26 (1973), 201-250.

2. V. V. GruSin, On a class of elliptic pseudodifferential operators that are degenerate 
on a submanifold, Mat. Sb. 84 (126) (1971), 163-195 = Math USSR Sb. 13 (1971), 155186. MR 44 \#860.

3. F. Trèves, Ovcyannicov theorem and hyperdifferential operators, Notas de Matemática, no. 46, Instituto de Matematica Pura e Aplicada, Conselho Nacional de Pesquisas, Rio de Janeiro, 1968. MR 44 \#7386.

4. L. Boutet De Monvel and F. Trèves, On a class of pseudodifferential operators with double characteristics, Invent. Math. 24 (1974), 1-34.

VIA VITTORIO VENETO 45, 65100, PESCARA, ITALY 